\title{
MODELLING OF CLASSICAL AND ROTARY INVERTED PENDULUM SYSTEMS - A GENERALIZED APPROACH
}

\author{
Slávka Jadlovská - Ján Sarnovský *
}

\begin{abstract}
The purpose of this paper is to present the design and program implementation of a general procedure which yields the mathematical model for a classical or rotary inverted pendulum system with an arbitrary number of pendulum links. Lagrange equations of the second kind with an integrated Rayleigh dissipation function are employed in model design, and the energetic balance relations, derived for the base and all pendulum links in a generalized (n-link) classical and rotary inverted pendulum system, are implemented in form of symbolic MATLAB functions and a MATLAB GUI application. The validity and accuracy of motion equations generated by the application are demonstrated by evaluating the open-loop responses of simulation models of classical double and rotary single inverted pendulum.
\end{abstract}

K e y w o r d s: inverted pendulum systems, automated mathematical modelling, Lagrange mechanics, symbolic MATLAB functions, classical double inverted pendulum, rotary single inverted pendulum

\section{INTRODUCTION}

Inverted pendulum systems represent a significant class of nonlinear underactuated mechanical systems which exhibit numerous problems present in industrial applications, such as a variety of external disturbances or nonlinear behaviors under different operation conditions [1], and have therefore assumed a prominent position among the test-bed systems for verification and practice of ideas emerging in control theory and robotics. Stabilization of a pendulum rod in the unstable upright position is considered a benchmark control problem which has been solved by attaching the pendulum to a base that moves in a controlled linear manner (classical inverted pendulum systems) or in a rotary manner in a horizontal plane (rotary inverted pendulum systems) [2-4].

In order to understand, predict, simulate, or control a physical system, a mathematical model of the system is generally required, usually in form of differential equations of motion [5]. It is only through complex mathematical and physical analysis that we can obtain motion equations which model the real system dynamics with sufficient accuracy [6]. For various examples of inverted pendulum systems, this analysis has been done ever since the system was introduced to feedback control community and the derived equations of motion are included in corresponding sources. Systems with a different number of pendulum links are standardly treated as independent units ( $e g[7]$ provides the motion equations for a single, double and triple pendulum system, derived independently of each other), which only permits the laborious and error-prone manual model derivation

For our research, we decided to focus on the obvious mutual analogy of the systems, which led to an important consequence: if a system of inverted pendula is ap- proached as an instance of a generalized system of inverted pendula (ie a system with n pendulum links), the respective steps of mathematical model derivation can be processed into an algorithm and implemented via symbolic software tools. The final result of this technique is a systematic means for generating the equations of motion, which provides a number of advantages over manual mathematical modelling we obtain a particularly precise approximation of the real system's dynamics and any factual or numeric errors which could arise during manual modelling are eliminated. Moreover, generalizations of this kind can be further extended to control algorithm design, and applied in real-time control of laboratory systems.

The goal of this paper is to enable automatic model generation for inverted pendulum systems by designing a general procedure which determines the motion equations for a system with an arbitrary number of pendula. The paper is organized as follows. Section 2 presents the respective steps of mathematical model derivation for generalized classical and rotary inverted pendulum systems, which form the core of the general procedure. Section 3 provides details of the program implementation of the procedure and proceeds to demonstrate its validity and accuracy using two example systems: classical double and rotary single inverted pendulum. In both cases, the simulation setup is built out of suitable function blocks from the Inverted Pendula Modeling and Control (IPMaC), a structured Simulink block library which was developed by the authors of this paper and provides complex software support for the analysis and control of classical and rotary inverted pendulum systems [2]. Finally, Section 4 concludes the paper with an evaluation of achieved results and some final remarks.

* Department of Cybernetics and Artificial Intelligence, Faculty of Electrical Engineering and Informatics, Technical University of Košice, Letná 9, 04200 Košice, Slovakia, slavka.jadlovska@tuke.sk, jan.sarnovsky@tuke.sk 


\section{MATHEMATICAL MODELLING OF GENERALIZED INVERTED PENDULUM SYSTEMS}

In this section, we will analyze the generalized ( $n$-link) classical and rotary inverted pendulum system using the Lagrangian formulation of mechanics [5]. This approach was chosen for several reasons: it is by definition suitable for generalizations, provides a more direct and less errorprone model design than eg force summation based on Newtons 2nd law; and the implementation of the method is straightforward with aid of symbolic software tools [8].

\subsection{Basic Preliminaries, Main Concepts and Definitions}

Let us define the generalized system of classical inverted pendula as a set of $n \geq 1$ rigid, homogenous, isothropic rods (pendulum rods or links) which are interconnected in joints and attached to a stable mechanism which allows for movement alongside a single axis (often referred to as a cart, moving carriage or wagon). Analogically, the generalized system of rotary inverted pendula is composed of $n \geq 1$ interconnected homogenous rods mounted on a rigid arm which rotates in a horizontal plane, perpendicular to the pendula [2]. In both cases, the system is underactuated because the number of actuators is lower than the number of system links: the only input (the force $F(t)$ acting upon the cart or the torque $M(t)$ applied on the rotary arm) is used to control the $n+1$ outputs of the system: cart position (in meters) or arm angle (in radians) and pendula angles (in radians) $[3,4]$.

Every system of $n$ inverted pendula is a multi-body composed of the base and the individual pendula, and thus has $n+1$ degrees of freedom. Any possible configuration of such a system can be uniquely defined by the following vector of generalized coordinates

$$
\boldsymbol{\theta}(t)=\left(\begin{array}{llll}
\theta_{0} & \theta_{1} & \ldots & \theta_{n}
\end{array}\right)^{\top}
$$

where $\theta_{0}(t)$ represents the position of the base (cart position for the system of classical inverted pendula, or arm angle for the system of rotary inverted pendula) and $\theta_{1}(t)$ to $\theta_{n}(t)$ stand for the angles of pendulum links.

According to the principle of virtual work, which was developed by Lagrange in 1788 and allows the transition from Newtonian to Lagrangian mechanics, a mechanical system is balanced if and only if the net virtual work $\delta W(t)$ due to all generalized forces is zero [5]

$$
\delta W(t)=\mathbf{Q}^{\top}(t) \delta \boldsymbol{\theta}(t)=0 .
$$

where $\mathbf{Q}(t)$ is a vector of generalized forces and $\delta \boldsymbol{\theta}(t)$ is a vector of virtual displacements along the corresponding generalized coordinates.

From the principle of virtual work, Euler-Lagrange equations of second kind can be determined, one for each generalized coordinate (ie degree of freedom of the system). The condensed vector form of the equations is [8]

$$
\frac{\mathrm{d}}{\mathrm{d} t}\left(\frac{\partial L(t)}{\partial \dot{\boldsymbol{\theta}}(t)}\right)-\frac{\partial L(t)}{\partial \boldsymbol{\theta}(t)}+\frac{\partial D(t)}{\partial \dot{\boldsymbol{\theta}}(t)}=\mathbf{Q}^{*}(t)
$$

where the Lagrange function (Lagrangian) $L(t)$ is defined as the difference between the kinetic $E_{K}$ and potential energy $E_{P}$ of the system

$$
L(t)=E_{K}(\boldsymbol{\theta}(t), \boldsymbol{\theta}(t))-E_{p}(\boldsymbol{\theta}(t)),
$$

the Rayleigh (dissipation) function $D(t)$ describes the viscous friction (dissipative properties)

$$
D(t)=D(\dot{\boldsymbol{\theta}}(t))
$$

and $\mathbf{Q}^{*}(t)$ is the vector of generalized external inputs applied on the system.

Only the dynamical behavior of the base is directly affected by the external input. The vector of generalized non-conservative external inputs is therefore defined as

$$
\mathbf{Q}^{*}=\left(\begin{array}{llll}
X(t) & 0 & \ldots & 0
\end{array}\right)^{\top}
$$

where $X(t)$ represents either the force $F(t)$ applied on the cart, or the torque $M(t)$ applied on the rotary arm.

To be able to determine $L(t)$ and $D(t)$, the total energy of a multi-body system must be specified as the sum of energies that correspond to the individual bodies it contains. Any system of $n$ inverted pendula is therefore characterized by relations

$$
\begin{gathered}
{ }^{n} E_{k}(t)=\sum_{i=0}^{n} E_{k i}(t), \quad{ }^{n} E_{p}(t)=\sum_{i=0}^{n} E_{p i}(t), \\
{ }^{n} D(t)=\sum_{i=0}^{n} D_{i}(t)
\end{gathered}
$$

where $E_{K i}(t), E_{P i}(t)$ and $D_{i}(t)$ respectively stand for the kinetic and potential energies, and the dissipative properties of each body. The process of derivation of motion equations that describe a selected system of inverted pendula has thus transformed into the determination of kinetic, potential and dissipation energies which relate to the base and all pendulum links and need to be expressed through the generalized coordinates.

In the following sections, we will derive the required physical relations and formulae that describe the energetic balances of the base and $i$-th pendulum in a system of $n$ inverted pendula. Both classical and rotary inverted pendulum systems will be dealt with. Indexing of all model parameters will obey the convention set by (1), where index 0 is assigned to the base and 1 to $n$ represent the individual pendulum links, starting with the link which is attached directly to the base. The nomenclature of numerical model parameters will therefore be as follows

$s_{x i}(t), s_{y i}(t), s_{z i}(t)$ - position (displacement) coordinates, $v_{x i}(t), v_{y i}(t), v_{z i}(t)$ - velocities in the directions of the axes,

$m_{i}(\mathrm{~kg})-$ mass of the base $(i=0)$ and the pendula $(i=1$ to $i=n)$,

$l_{i}(\mathrm{~m})$ - length of the arm $(i=0)$ or the $i$-th pendulum $(i=1$ to $i=n)$,

$g\left(\mathrm{~ms}^{-2}\right)-$ acceleration due to gravity $\left(g=9.81 \mathrm{~ms}^{-2}\right.$ will be used),

$\delta_{i}\left(\mathrm{kgs}^{-1}\right) /\left(\mathrm{kgm}^{2} \mathrm{~s}^{-1}\right)$ - friction coefficient of the base against the surface $(i=0) /$ damping constant related to the pivot point of $i$-th pendulum $(i=1$ to $i=n)$. 


\subsection{Generalized System Of Classical Inverted Pendula}

The definition of a generalized classical inverted pendulum system will now be expanded. Throughout the derivation process, let us assume that (Fig. 1):

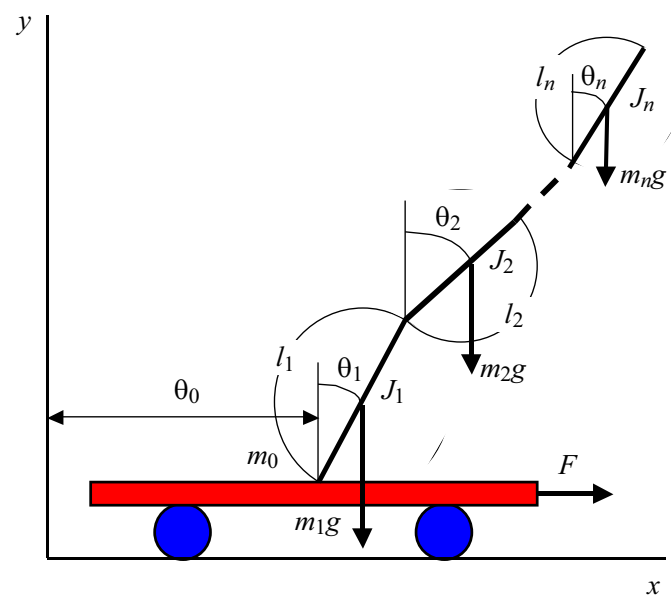

Fig. 1. System of $n$ classical inverted pendula - scheme and basic nomenclature

(1) the modelling takes place in a planary (two-dimensional) coordinate system, ie all motion is bound to the $x y$-plane with the cart moving along a line identical to the $x$-axis, which simultaneously represents the projection of the zero potential energy level into the $x y$-plane;

(2) the value of every pendulum angle is determined clockwise from the vertical upright position, which is parallel to the $y$-axis;

(3) the only input of the system is the force applied on the cart in the direction parallel to the $x$-axis and to the cart itself.

\section{Energetic balance of the cart}

Since the cart is assumed to be moving in a linear manner, we can provide its mathematical description using a single spatial dimension. If we identify it with the $x$-axis, the only position coordinate will be denoted as $s_{x 0}(t)=\theta_{0}(t)$, which means (see assumptions) that the potential energy of the cart

$$
E_{P 0}(t)=0
$$

The kinetic energy and the dissipation function both depend on the cart velocity $v_{x 0}(t)=\dot{\theta}_{0}(t)$

$$
\begin{aligned}
E_{K 0}(t) & =\frac{1}{2} m_{0} v_{x 0}^{2}(t)=\frac{1}{2} m_{0} \dot{\theta}_{0}^{2}(t), \\
D_{0}(t) & =\frac{1}{2} \delta_{0} v_{x 0}^{2}(t)=\frac{1}{2} \delta_{0} \dot{\theta}_{0}^{2}(t) .
\end{aligned}
$$

\section{Energetic balance of the $i$-th pendulum}

Let us suppose that the whole mass of a pendulum rod is concentrated in its center of gravity (CoG) which is identical to the geometrical center of the rod in the distance of $l_{i} / 2$ from the pivot point. The coordinates of the CoG of $i$-th pendulum rod are therefore expressed as (Fig. 1)

$$
\left(\begin{array}{c}
s_{x i}(t) \\
s_{y i}(t)
\end{array}\right)=\left(\begin{array}{c}
\theta_{0}(t)+\sum_{k=1}^{i} l_{k} \sin \theta_{k}(t)-\frac{l_{i}}{2} \sin \theta_{i}(t) \\
\sum_{k=1}^{i} l_{k} \cos \theta_{k}(t)-\frac{l_{i}}{2} \cos \theta_{i}(t)
\end{array}\right)
$$

and the velocity components in the directions of the axes equal

$$
\begin{aligned}
& \left(\begin{array}{c}
v_{x i}(t) \\
v_{y i}(t)
\end{array}\right)=\frac{\mathrm{d}}{\mathrm{d} t}\left(\begin{array}{c}
s_{x i}(t) \\
s_{y i}(t)
\end{array}\right)= \\
& \left(\begin{array}{c}
\dot{\theta}_{0}(t)+\sum_{k=1}^{n} l_{k} \dot{\theta}_{k}(t) \cos \theta_{k}(t)-\frac{l_{i}}{2} \dot{\theta}_{i}(t) \cos \theta_{i}(t) \\
-\sum_{k=1}^{i} l_{k} \dot{\theta}_{k}(t) \sin \theta_{k}(t)+\frac{l_{i}}{2} \dot{\theta}_{i}(t) \sin \theta_{i}(t)
\end{array}\right)_{(12}
\end{aligned}
$$

The potential energy of the $i$-th pendulum is determined by the height of its CoG above the $x$-axis

$$
E_{P i}(t)=m_{i} g s_{y i}(t)=m_{i} g\left(\sum_{k=1}^{i} l_{k} \cos \theta_{k}(t)-\frac{l_{i}}{2} \cos \theta_{i}(t)\right)
$$

and the kinetic energy of each pendulum is given as a sum of two components that describe the pendulum's translational and rotary motion, [9]

$$
E_{K i}(t)=\frac{1}{2} m_{i} v_{i}^{2}(t)+\frac{1}{2} J_{T i} \dot{\theta}_{i}^{2}(t)
$$

where $J_{n}=m_{i} L_{i}^{2} / 12$ represents the pendulum's moment of inertia with respect to the CoG and

$$
v_{i}(t)=\sqrt{v_{x i}^{2}(t)+v_{y i}^{2}(t)}
$$

is the magnitude of the pendulum's translational velocity.

The dissipative properties of the $i$-th pendulum are quadratically dependent on the angular velocities of pendulum links marked as $i$ and $i-1$

$$
D_{i}(t)=\frac{1}{2} \delta_{i}\left(\dot{\theta}_{i}(t)-\dot{\theta}_{i-1}(t)\right)^{2}
$$

and

$$
D_{i}(t)=\frac{1}{2} \delta_{i} \dot{\theta}_{i}^{2}(t)
$$

for $n=1$ (single inverted pendulum system). 


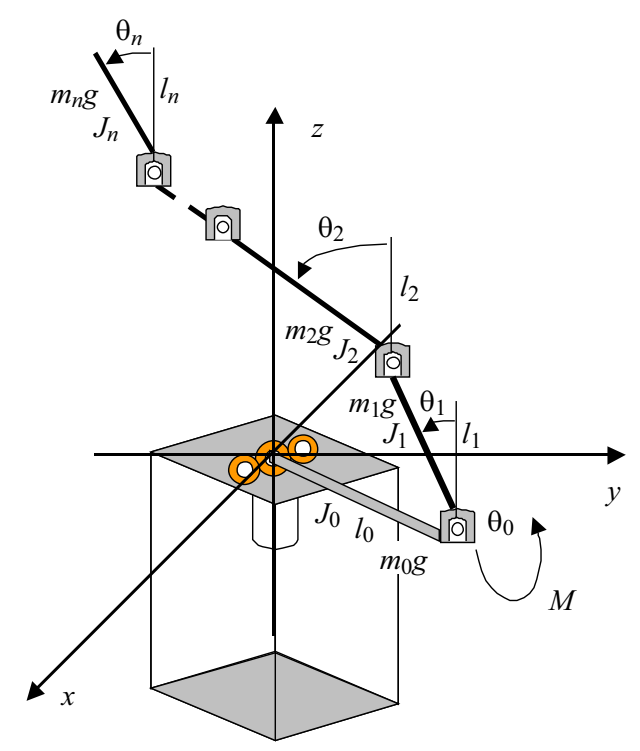

Fig. 2. System of $n$ rotary inverted pendula - scheme and basic nomenclature

\subsection{Generalized System of Rotary Inverted Pen- dula}

To expand the definition of the generalized rotary inverted pendulum system (Fig. 2), the following was assumed:

(1) all modelling is bound to a standard right-handed three-dimensional (Cartesian) coordinate system; the arm rotates in a horizontal (parallel to $x y$ ) plane which is identical to the zero potential energy level;

(2) the rotary motion of each pendulum takes place in a vertical plane that is always perpendicular to both the horizontal arm plane and the arm itself; both the upright and downward (hanging) position are parallel with the $z$-axis and the value of every pendulum angle is determined clockwise from the upright position;

(3) the input which actuates the system is the torque applied on the rotary arm.

The balance relations for rotary inverted pendulum systems are analogical in many aspects with the relations (8-17), derived in the previous section for classical inverted pendulum systems.

\section{Energetic balance of the rotary arm}

It was assumed at the beginning that the potential energy of the rotary plane, and hence of the arm, would be zero

$$
E_{P 0}(t)=0 \text {. }
$$

Two spatial coordinates are therefore sufficient to provide a complete description of the arm dynamics. The coordinates of the arm's CoG are specified as (Fig. 2)

$$
\left(\begin{array}{c}
s_{x 0}(t) \\
s_{y 0}(t)
\end{array}\right)=\frac{l_{0}}{2}\left(\begin{array}{c}
\sin \theta_{0}(t) \\
\cos \theta_{0}(t)
\end{array}\right)
$$

and the velocities in the directions of the axes respectively equal

$$
\left(\begin{array}{c}
v_{x 0}(t) \\
v_{y 0}(t)
\end{array}\right)=\frac{l_{0} \dot{\theta(t)_{0}}}{2}\left(\begin{array}{c}
\cos \theta_{0}(t) \\
-\sin \theta_{0}(t)
\end{array}\right)
$$

The kinetic energy of the arm consists of a translational and a rotary component

$$
E_{K 0}(t)=\frac{1}{2} m_{0} v_{0}^{2}(t)+\frac{1}{2} J_{T 0} \dot{\theta}_{0}^{2}(t)
$$

where $J_{T 0}=m_{0} l_{0}^{2} / 12$ is the arm's moment of inertia with respect to the center of gravity, and the arm's translational velocity has the magnitude of

$$
v_{0}(t)=\sqrt{v_{x 0}^{2}(t)+v_{y 0}^{2}(t)} .
$$

Inserting (20) into (22) and then into (21) yields

$$
E_{K 0}(t)=\frac{1}{2} J_{0} \dot{\theta}_{0}^{2}(t)
$$

where $J_{0}=m_{0} l_{0}^{2} / 3$ is the moment of inertia with respect to the rotary axis.

Finally, the dissipative properties of the arm are given as

$$
D_{0}(t)=\frac{1}{2} \delta_{0} \dot{\theta}_{0}^{2}(t) .
$$

Energetic balance of $i$-th pendulum

Assuming that every pendulum rotates in a vertical plane, the coordinates of the CoG for the $i$-th pendulum are

$$
\begin{aligned}
& \left(\begin{array}{c}
s_{x i}(t) \\
s_{y i}(t) \\
s_{z i}(t)
\end{array}\right)= \\
& \left(\begin{array}{c}
l_{0} \sin \theta_{0}(t)+\left(\sum_{k=1}^{i} l_{k} \sin \theta_{k}(t)-\frac{l_{i}}{2} \sin \theta_{i}(t)\right) \cos \theta_{0}(t) \\
l_{0} \cos \theta_{0}(t)-\left(\sum_{k=1}^{i} l_{k} \sin \theta_{k}(t)-\frac{l_{i}}{2} \sin \theta_{i}(t)\right) \sin \theta_{0}(t) \\
\sum_{k=1}^{i} l_{k} \cos \theta_{k}(t)-\frac{l_{i}}{2} \cos \theta_{i}(t)
\end{array}\right)
\end{aligned}
$$

and the velocity components of the pendulum CoG in the directions of the $x, y$ and $z$ axes become

$$
\begin{aligned}
& \left(\begin{array}{c}
v_{x i}(t) \\
v_{y i}(t) \\
v_{z i}(t)
\end{array}\right)=\frac{\mathrm{d}}{\mathrm{d} t}\left(\begin{array}{c}
s_{x i}(t) \\
s_{y i}(t) \\
s_{z i}(t)
\end{array}\right)= \\
& \left(\begin{array}{c}
l_{0} \dot{\theta}_{0}(t) \cos \theta_{0}(t)+\sum_{k=1}^{i} l_{k} \dot{\theta}_{k}(t) \cos \theta_{k}(t) \cos \theta_{0}(t) \\
-\sum_{k=1}^{i} l_{k} \dot{\theta}_{0}(t) \sin \theta_{k}(t) \sin \theta_{0}(t)-\frac{l_{i}}{2} \dot{\theta}_{i}(t) \cos \theta_{i}(t) \cos \theta_{0}(t) \\
+\frac{l_{i}}{2} \dot{\theta}_{0}(t) \sin \theta_{i}(t) \sin \theta_{0}(t) \\
-l_{0} \dot{\theta}_{0}(t) \sin \theta_{0}(t)-\sum_{k=1}^{i} l_{k} \dot{\theta}_{k}(t) \cos \theta_{k}(t) \sin \theta_{0}(t) \\
-\sum_{k=1}^{i} l_{k} \dot{\theta}_{0}(t) \sin \theta_{k}(t) \cos \theta_{0}(t)+\frac{l_{i}}{2} \dot{\theta}_{i}(t) \cos \theta_{i}(t) \sin \theta_{0}(t) \\
+\sum_{k=1}^{n} l_{k} \dot{\theta}_{k}(t) \sin \theta_{k}(t)+\frac{l_{i}}{2} \dot{\theta}_{i}(t) \sin \theta_{i}(t) \cos \theta_{0}(t)
\end{array}\right)
\end{aligned}
$$


The potential energy of the $i$-th pendulum is determined by the height of the pendulum CoG above the rotational plane of the arm

$$
E_{P i}(t)=m_{i} g s_{z i}(t)=m_{i} g\left(\sum_{k=1}^{i} l_{k} \cos \theta_{k}(t)-\frac{l_{i}}{2} \cos \theta_{i}(t)\right)
$$

and the kinetic energy of the $i$-th pendulum is once again determined as a sum of a translational and rotary component (14). While the pendulum's moment of inertia with respect to the CoG remains equal to $J_{T i}=m_{i} l_{i}^{2} / 12$, the translational velocity of the pendulum has the magnitude of

$$
v_{i}(t)=\sqrt{v_{x i}^{2}(t)+v_{y i}^{2}(t)+v_{z i}^{2}(t)} .
$$

The dissipative properties of the pendula are described as (16); or (17), if $n=1$.

\section{GENERAL PROCEDURE - PROGRAM IMPLEMENTATION AND APPLICATION}

Based on the theoretical background presented in 2.1 as well as the physical formulae derived in 2.2 and 2.3 , we constructed a pair of general algorithmic procedures which lead to the equations of motion for an arbitrary classical or rotary system of inverted pendula. With aid of the Symbolic Math Toolbox, we successfully implemented both procedures as MATLAB functions (invpenderiv.m for classical, rotinvpenderiv.m. for rotary), which produce the equations in the simplified and rearranged form, equivalent to the most likely form obtained by manual derivation. In both cases, the number of pendulum links needs to be specified as the function parameter and the physically significant steps of derivation process can be tracked in the command window output of the function. Finally, we developed a MATLAB application with graphical user interface, the Inverted Pendula Model Equation Derivator, which provides a comfortable and user-friendly access to both functions: the user is only required to select the desired type of system (classical/rotary) and the number of pendulum links. An example preview of the Derivator window is listed in [2] together with generated motion equations for a specified inverted pendulum system.

To demonstrate the functionality of presented general procedures, mathematical models of classical double and rotary single inverted pendulum system were generated by the Derivator and will be presented below, rearranged into the standard (minimal ODE-ordinary differential equation) form [10]

$$
\mathbf{M}(\boldsymbol{\theta}(t)) \ddot{\boldsymbol{\theta}}(t)+\mathbf{N}(\boldsymbol{\theta}(t), \dot{\boldsymbol{\theta}}(t)) \dot{\boldsymbol{\theta}}(t)+\mathbf{P}(\boldsymbol{\theta}(t))=\mathbf{V}(t)
$$

where $\mathbf{M}(\boldsymbol{\theta}(t))$ is the inertia matrix, $\mathbf{N}(\boldsymbol{\theta}(t), \dot{\boldsymbol{\theta}}(t))$ describes the influence of centrifugal and Coriolis forces, $\mathbf{P}(\boldsymbol{\theta}(t))$ accounts for gravity forces and $\mathbf{V}(t)$ is the input vector, equivalent to (6). By comparing the obtained results to those listed in the referenced works, and by evaluating the open-loop responses of corresponding simulation models, it will next be assessed whether the generated mathematical models can be considered as valid and accurate for control design purposes.

\subsection{Case Study - Classical Double Inverted Pendulum System}

The classical double (two-link) inverted pendulum system is composed of a pair of rigid, homogenous pendulum rods which are interconnected in a joint and one of them is attached to the cart [3]. The force input $F(t)$ acting upon the cart is used to control the three degrees of freedom of the system: cart position $\theta_{0}(t)$ in $\mathrm{m}$, lower pendulum angle $\theta_{1}(t)$ in rad, and upper pendulum angle $\theta_{2}(t)$ in rad, (see Fig. 3).

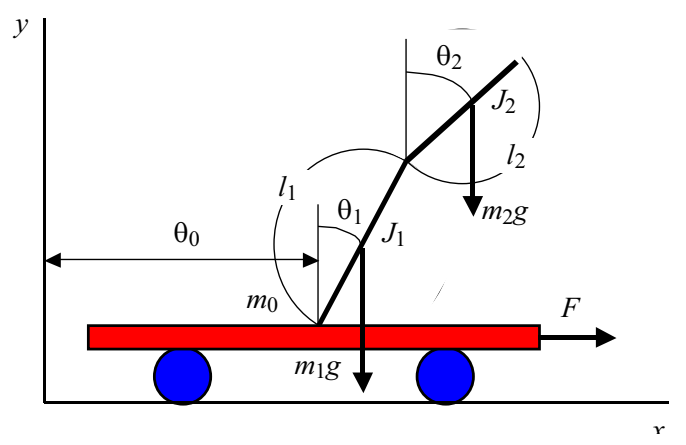

Fig. 3. Classical double inverted pendulum system - scheme and basic nomenclature

Using the Derivator, the mathematical model of the system was generated in form of three second-order nonlinear differential equations which describe the dynamic behavior of the cart, lower and upper pendulum respectively. After the rearrangement into (29), the model assumed the form

$$
\begin{aligned}
& \left(\begin{array}{cc}
m_{0}+m_{1}+m_{2} & \left(\frac{1}{2} m_{1} l_{1}+m_{2} l_{1}\right) \cos \theta_{1}(t) \\
\left(\frac{1}{2} m_{1} l_{1}+m_{2} l_{1}\right) \cos \theta_{1}(t) & J_{1}+m_{2} l_{1}^{2} \\
\frac{1}{2} m_{2} l_{2} \cos \theta_{2}(t) & \frac{1}{2} m_{2} l_{1} l_{2} \cos \left(\theta_{1}(t)-\theta_{2}(t)\right)
\end{array}\right. \\
& \left.\begin{array}{c}
\frac{1}{2} m_{2} l_{2} \cos \theta_{2}(t) \\
\frac{1}{2} m_{2} l_{1} l_{2} \cos \left(\theta_{1}(t)-\theta_{2}(t)\right) \\
J_{2}
\end{array}\right)\left(\begin{array}{l}
\ddot{\theta}_{0}(t) \\
\ddot{\theta}_{1}(t) \\
\ddot{\theta}_{2}(t)
\end{array}\right)+ \\
& \left(\begin{array}{cc}
\delta_{0} & -\left(\frac{1}{2} m_{1} l_{1}+m_{2} l_{1}\right) \dot{\theta}_{1}(t) \sin \theta_{1}(t) \\
\frac{1}{2} m_{1} l_{1} \cos \theta_{1}(t) & \delta_{1}+\delta_{2} \\
\frac{1}{2} m_{2} l_{2} \cos \theta_{2}(t) & -\delta_{2}-\frac{1}{2} m_{2} l_{1} l_{2} \dot{\theta}_{2}(t) \sin \left(\theta_{1}(t)-\theta_{2}(t)\right)
\end{array}\right. \\
& \left.\begin{array}{c}
\frac{1}{2} m_{2} l_{2} \cos \theta_{2}(t) \\
-\delta_{2}-\frac{1}{2} m_{2} l_{1} l_{2} \dot{\theta}_{2}(t) \sin \left(\theta_{1}(t)-\theta_{2}(t)\right) \\
\delta_{2}
\end{array}\right)\left(\begin{array}{c}
\dot{\theta}_{0}(t) \\
\dot{\theta}_{1}(t) \\
\dot{\theta}_{2}(t)
\end{array}\right)+ \\
& \left(\begin{array}{c}
0 \\
-\left(\frac{1}{2} m_{1}+m_{2}\right) g l_{1} \sin \theta_{1}(t) \\
-\frac{1}{2} m_{2} g l_{2} \sin \theta_{2}(t)
\end{array}\right)=\left(\begin{array}{c}
F(t) \\
0 \\
0
\end{array}\right)
\end{aligned}
$$


where $m_{0}$ is the cart mass, $m_{1}, m_{2}$ are the masses of the lower and upper pendulum respectively, $l_{1}, l_{2}$ are the respective lengths of the pendula, $\delta_{0}$ is the friction coefficient of the cart against the rail, $\delta_{1}, \delta_{2}$ are the damping constants in the joints of the pendula, $J_{1}=m_{1} l_{1}^{2} / 3=$ $J_{T 1}+m_{1}\left(l_{1} / 2\right)^{2}, J_{2}=m_{2} l_{2}^{2} / 3=J_{T 2}+m_{2}\left(l_{2} / 2\right)^{2}$ are the moments of inertia of the pendula with respect to their pivot points and $F(t)$ is the force induced on the cart.

It was discovered that in most cases, the generated motion equations of the system (30) are identical to manually derived equations in referenced works, provided the latter were derived correctly (compare (30) to the classical double inverted pendulum model in $[7,10,11])$. Apart from the neglected friction, the only significant difference noticed was the plus sign at the potential energy terms $\left(e g m_{2} g l_{2} \sin \theta_{2}(t) / 2\right.$ instead of $\left.-m_{2} g l_{2} \sin \theta_{2}(t) / 2\right)$, which corresponds to an initial assumption that the pendulum angle is determined against the downward position.

The IPMaC block library contains a library block Classical Double Inverted Pendulum (CDIP) which implements the motion equations (30) derived above. The open-loop dynamic behavior of the system was verified as a response of the $C D I P$ block to a force impulse with an amplitude of $0.4 \mathrm{~N}$ and duration of $1 \mathrm{~s}$, starting with all pendula in the upright position. The numeric parameters of the simulation were chosen as follows: $m_{0}=0.3 \mathrm{~kg}$, $m_{1}=m_{2}=0.275 \mathrm{~kg}, l_{1}=l_{2}=0.5 \mathrm{~m}, \delta_{0}=0.3 \mathrm{kgs}^{-1}$, $\delta_{1}=0.1 \mathrm{kgm}^{2} \mathrm{~s}^{-1}, \delta_{2}=0.1 \mathrm{kgm}^{2} \mathrm{~s}^{-1}$. The time behavior of the cart position and the freefall and stabilization of both pendula is depicted in Fig. 4.
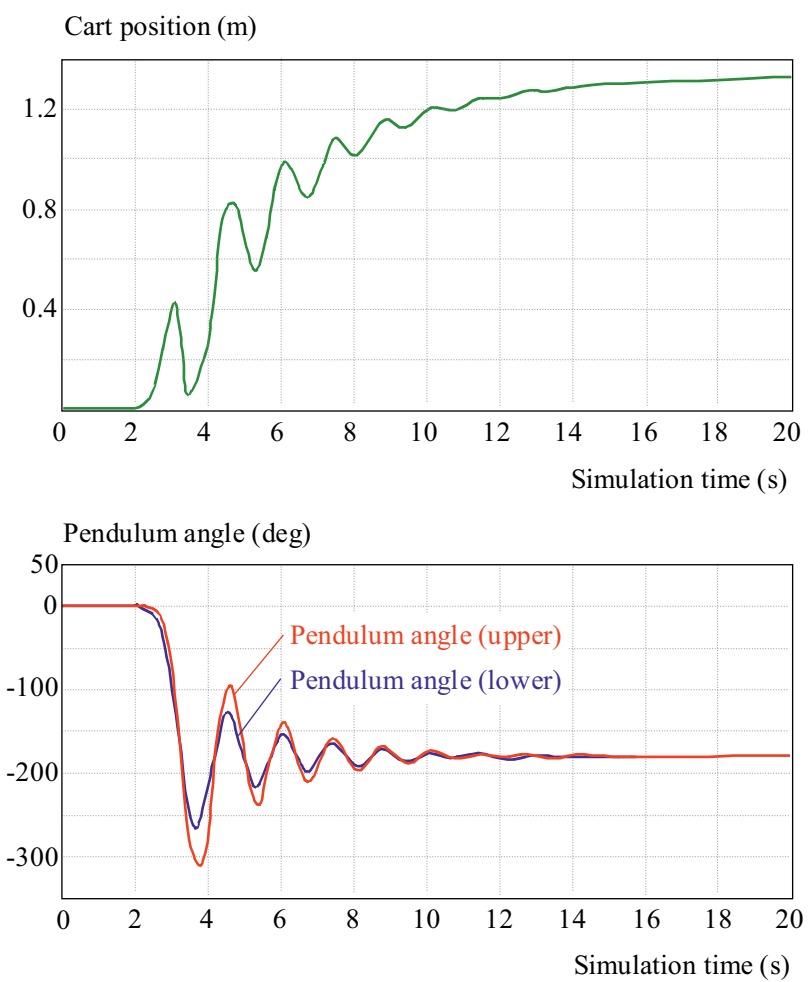

Fig. 4. Classical double inverted pendulum system time behavior - cart position and pendula angles
Reasonable behavior of the open-loop response of the CDIP simulation model (damped oscillatory transient state, system reaching the stable equilibrium point with all pendula pointing downward, visible backward impact of the pendula on the base) means that the simulation model based on the generated motion equations can be considered accurate enough to serve as a reliable testbed system for the verification of linear and nonlinear control algorithms.

\subsection{Case Study - Rotary Single Inverted Pendu- lum System}

The rotary single inverted pendulum system is composed of a homogenous pendulum rod attached to an arm which is free to rotate in a horizontal plane [4]. The torque $M(t)$ applied on the arm is used to control the two degrees of freedom of the system: arm angle $\theta_{0}(t)$ in rad, and pendulum angle $\theta_{1}(t)$ in rad (see Fig. 5). The system was introduced to feedback control community in 1992 by Katsuhisa Furuta, Professor at the Tokyo Institute of Technology, and hence it is often referred to as the Furuta pendulum [12].

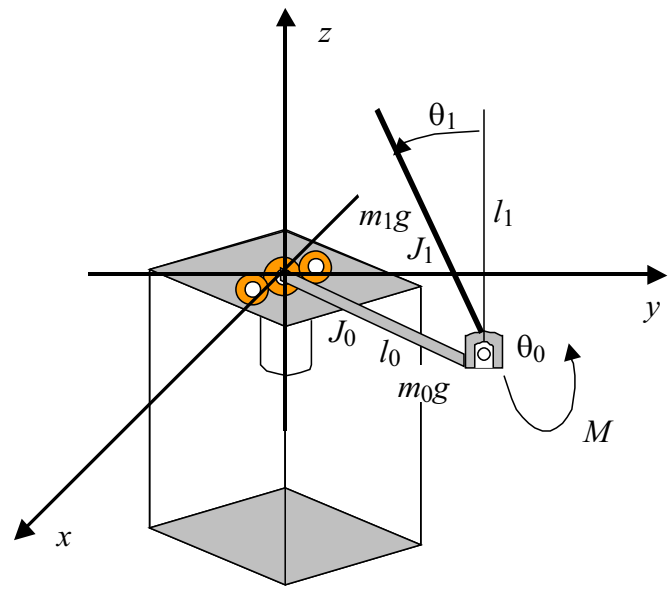

Fig. 5. Rotary single inverted pendulum system scheme and basic nomenclature

The mathematical model of the system is composed of two second-order nonlinear differential motion equations which respectively describe the rotary arm and the pendulum. The standard minimal form of the model generated by the Derivator is

$$
\begin{gathered}
\left(\begin{array}{cc}
J_{0}+m_{1} l_{0}^{2}+\frac{1}{4} m_{1} l_{1}^{2} \sin ^{2} \theta_{1}(t) & \frac{1}{2} m_{1} l_{0} l_{1} \cos \theta_{1}(t) \\
\frac{1}{2} m_{1} l_{0} l_{1} \cos \theta_{1}(t) & J_{1}
\end{array}\right)\left(\begin{array}{c}
\ddot{\theta}_{0}(t) \\
\ddot{\theta}_{1}(t)
\end{array}\right) \\
+\left(\begin{array}{cc}
\delta_{0}+\frac{1}{4} m_{1} l_{1}^{2} \dot{\theta}_{1}(t) \sin 2 \theta_{1}(t) & -\frac{1}{2} m_{1} l_{0} l_{1} \dot{\theta}_{1}(t) \sin \theta_{1}(t) \\
-\frac{1}{8} m_{1} l_{1}^{2} \dot{\theta}_{0}(t) \sin 2 \theta_{1}(t) & \delta_{1}
\end{array}\right) \\
\quad \times\left(\begin{array}{c}
\dot{\theta}_{0}(t) \\
\dot{\theta}_{1}(t)
\end{array}\right)+\left(\begin{array}{c}
0 \\
-\frac{1}{2} m_{1} g l_{1} \sin \theta_{1}(t)
\end{array}\right)=\left(\begin{array}{c}
M(t) \\
0
\end{array}\right)
\end{gathered}
$$

where $m_{0}, m_{1}$ stand for the masses of the arm and the pendulum, $l_{0}, l_{1}$ are their respective lengths, $\delta_{0}$, 

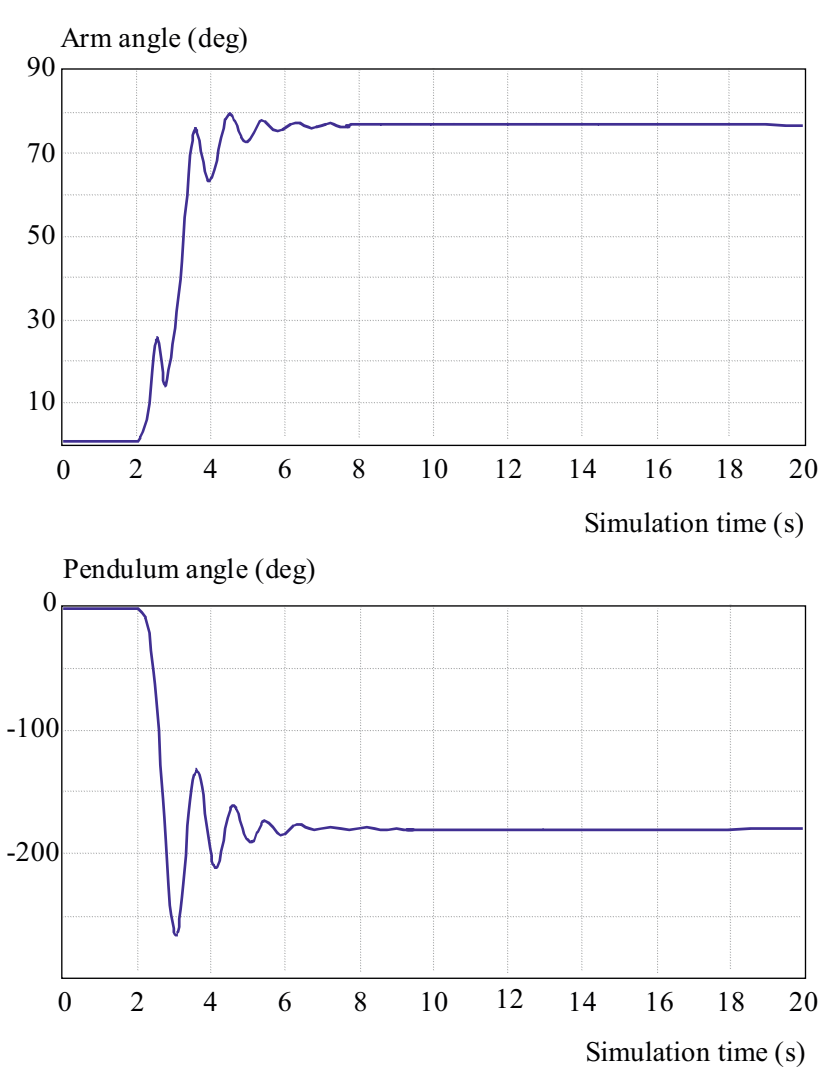

Fig. 6. Rotary single inverted pendulum system time behavior cart position and pendulum angle

$\delta_{1}$ are the damping constants in the joints of the arm and pendulum, $J_{0}=m_{0} l_{0}^{2} / 3=J_{T 0}+m_{0}\left(l_{0} / 2\right)^{2}$, and $J_{1}=m_{1} l_{1}^{2} / 3=J_{T 1}+m_{1}\left(l_{1} / 2\right)^{2}$ are the moments of inertia of the arm and pendulum with respect to their pivot points and $M(t)$ is the input torque applied upon the rotary arm.

Contrary to the previous case, a number of differences were noticed between the generated mathematical model (31) and the models in referenced works. Most of these can be attributed to the choice of coordinate system (right- or left-handed) and the orientation of the angles (clockwise/anticlockwise). Also, authors often simplify the design by assuming that the pendulum is rotating in a constant plane $[13,14]$ or neglect the rotary motion of the arm altogether [15]. In this paper, it was assumed that the actual plane the pendulum is rotating in is different in every instant, which brings additional complexity and high accuracy to the generated model.

The generated motion equations were implemented into the IPMaC in form of the Rotary Single Inverted Pendulum (RSIP) library block, and the open-loop dynamic behavior of the system was verified in a simulation experiment in which the $R S I P$ block was actuated from an initial upright position of the pendulum by a torque impulse of $0.4 \mathrm{Nm}$ lasting $1 \mathrm{~s}$. The numeric parameters of the simulated system were chosen to be: $m_{0}=0.5 \mathrm{~kg}$, $m_{1}=0.275 \mathrm{~kg}, l_{0}=0.6 \mathrm{~m}, l_{1}=0.5 \mathrm{~m}, \delta_{0}=0.3 \mathrm{kgs}^{-1}$, $\delta_{1}=0.011458 \mathrm{kgm}^{2} \mathrm{~s}^{-1}$.
The similarity between Fig. 4 and Fig. 6 points out that the dynamical behavior of classical and rotary inverted pendulum systems is closely related. The openloop response of the $R S I P$ simulation model was shown to be correct compared to generally known empirical observations of pendula behavior, and sufficient accuracy of the simulation model was confirmed.

\section{CONCLUSION}

The purpose of this paper was to introduce a unifying approach to the problem of mathematical model derivation for classical or rotary inverted pendulum systems with an arbitrary number of pendulum links. By means of extensive physical analysis, general algorithmic procedures were designed which determine the Euler-Lagrange equations for the base and every pendulum in a generalized system of $\mathrm{n}$ classical or rotary inverted pendula. The procedures were subsequently implemented as MATLAB functions which generate the motion equations for a user-chosen classical or rotary inverted pendulum system. Practical importance of symbolic mathematical software, represented in MATLAB by Symbolic Math Toolbox, was demonstrated during the implementation. A GUI application was also developed to provide a user-friendly, intuitive interface to both procedures.

Classical double and rotary single inverted pendulum system were selected as example systems to test the proposed modelling method. The validity and accuracy of mathematical models generated by the above application was verified by comparing the obtained results to those listed in the resources, and by evaluating the open-loop responses of corresponding simulation models. Pre-prepared function blocks from the custom-designed Simulink block library, Inverted Pendula Modeling and Control, were used in the simulation experiments. The outcome of both approaches was highly satisfactory: all possible deviations were justified by the laws of mechanics and it was shown that the dynamic behavior of all models is in agreement with the empirically observed facts about the pendula movement.

The results of this paper allow the control engineer to effortlessly obtain a highly accurate, error-free mathematical model of a selected inverted pendulum system, which simplifies the process of model-based design and verification of linear and nonlinear control methods for nonlinear mechanical systems. Moreover, the readily available collection of mathematical and simulation models of inverted pendulum systems provides the testbed model basis to explore the properties of underactuated nonlinear systems, mechatronic systems, hybrid automata and other concepts. Finally, the developed application can be viewed as a contribution to modelling and control education in several ways: students improve their modelling skills by testing their step-by-step results against a valid model, and estimate the effect of different control approaches in simulation before moving on to a laboratory model. 


\section{Acknowledgements}

This work has been supported by the Scientific Grant Agency of Slovak Republic under project No 1/0286/11 Dynamic Hybrid Architectures of the Multiagent Network Control Systems.

\section{REFERENCES}

[1] BAKER, G. J.-BLACKBURN, J. A.: The Pendulum: a Case Study in Physics, Oxford University Press, New York, 2005.

[2] JADLOVSKÁ, S.-SARNOVSKÝ, J. : An Extended Simulink Library for Modeling and Control of Inverted Pendula Systems, Proc. of the Int. Conf. Technical Computing Prague 2011, November 8, 2011, Prague, Czech Republic.

[3] JADLOVSKÁ, S.-SARNOVSKÝ, J.: Classical Double Inverted Pendulum - a Complex Overview of a System, Proc. of the IEEE 10th Int. Symposium on Applied Machine Intelligence and Informatics - SAMI 2012, January 26-28, 2012 Herl'any, Slovakia.

[4] JADLOVSKÁ, S.-SARNOVSKÝ, J. : A Complex Overview of the Rotary Single Inverted Pendulum System, Proc. of the 9th Int. Conf. - ELEKTRO 2012, May 21-22, 2012, Žilina - Rajecké Teplice, Slovakia, pp. 305-310.

[5] NOSKIEVIČ, J. : Modeling and Identification of Systems (Modelování a identifikace systémů), Montanex, Ostrava. (in Czech)

[6] ROUBAL, J.-HUŠEK, P. et al: Control Technology in Examples (Regulační technika v př́kladech), BEN technická literatura, Brno. (in Czech)

[7] SCHLEGEL, M.-MEŠŤÁNEK, J.: Limitations on the Inverted Pendula Stabilizability According to Sensor Placement, Proc. of the 16th Int. Conf. on Process Control, June 11-14, 2007, Štrbské Pleso, Slovakia.

[8] HALLIDAY, D.-RESNICK, R.-WALKER, J. : Fundamentals of Physics, Extended 7th edition, Wiley, June 16, 2004.

[9] HAJKO, V.-DANIEL-SZABÓ, J.: Fundamentals of physics (Základy fyziky), VEDA Slovak Academy of Sciences, Bratislava, 1980. (in Slovak)

[10] BOGDANOV, A.: Optimal Control of a Double Inverted Pendulum on a Cart, Technical Report CSE-04-006, OGI School of Science and Engineering, OHSU, 2004.

[11] DEMIRCI, M.: Design of Feedback Controllers for a Linear System with Applications to Control of a Double Inverted Pendulum, Int. Journal of Computational Cognition 2 No. 1 (2004), $65-84$.
[12] FURUTA, K.-YAMAKITA, M.-KOBAYASHI, S.: Swing up Control of Inverted Pendulum, Proc. of the Int. Conf. on Industrial Electronics, Control and Instrumentation (IECON91), Oct 28-Nov 1, 1991, Kobe, Japan.

[13] ERNEST, P.-HORÁČEK, P. : Algorithms for Control of a Rotating Pendulum, Proc. of the 19th IEEE Mediterranean Conf. on Control and Aut (MED'11), Corfu, Greece, 2011.

[14] KATS, C. J. A. : Nonlinear Control of a Furuta Rotary Inverted Pendulum, DCT Report, No. 2004:69, 2004.

[15] REX Controls s r.o., Rotary Inverted Pendulum FPM-210/211. User Manual (Rotační inverzní kyvadlo FPM-210/211. Užívatelská příručka), 2010. (in Czech)

Received 13 July 2012

Slávka Jadlovská born in 1988 in Košice, Slovakia, graduated in 2011 (MSc) with distinction at the Department of Cybernetics and Artificial Intelligence of the Faculty of Electrical Engineering and Informatics at Technical University in Košice. She is currently continuing her studies at the same department as a $\mathrm{PhD}$ student. Her dissertation research is focused on the hybrid methods of control for dynamic systems. In addition, she investigates issues related with modelling of under-actuated mechatronic systems, optimal and predictive control design for hybrid systems, and the advanced application of MATLAB toolboxes in modelling and control. So far, she has authored several scientific articles published in international conference proceedings.

Ján Sarnovský born in 1945, obtained his MSc degree in Technical Cybernetics on the Faculty of Electrical Engineering of the Slovak Technical University Bratislava, in 1968. He defended his dissertation thesis in the field of Automation and Control in 1980 at the same University; his thesis title was Control of Large Scale Systems Using Hybrid Computer Systems. Since 1969 he has been working at Faculty of Electrical Engineering and Informatics Technical University in Koice as a Associate Assistant, since 1980 as Associate Professor and since 1993 as a full Professor. Since 1985 he has been working as a tutor with the Department of Cybernetics and Artificial Intelligence. His scientific research focuses on the large scale systems and multiagent systems in control of the large scale systems. He has authored a number of scientific articles and contributions published in the journals and international conference proceedings. He is also an author of several monographs. 